\title{
A Process Model for Dashboard Onboarding
}

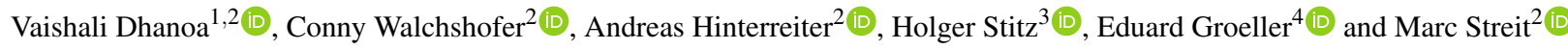 \\ ${ }^{1}$ Pro2Future GmbH, Austria \\ ${ }^{2}$ Johannes Kepler University Linz, Linz, Austria \\ ${ }^{3}$ datavisyn $\mathrm{GmbH}$, Linz, Austria \\ ${ }^{4}$ TU Wien \& VRVis Research Center, Austria
}

\begin{abstract}
Dashboards are used ubiquitously to gain and present insights into data by means of interactive visualizations. To bridge the gap between non-expert dashboard users and potentially complex datasets and/or visualizations, a variety of onboarding strategies are employed, including videos, narration, and interactive tutorials. We propose a process model for dashboard onboarding which formalizes and unifies such diverse onboarding strategies. Our model introduces the onboarding loop alongside the dashboard usage loop. Unpacking the onboarding loop reveals how each onboarding strategy combines selected building blocks of the dashboard with an onboarding narrative. Specific means are applied to this narration sequence for onboarding, which results in onboarding artifacts that are presented to the user via an interface. We concretize these concepts by showing how our process model can be used to describe a selection of real-world onboarding examples. Finally, we discuss how our model can serve as an actionable blueprint for developing new onboarding systems.
\end{abstract}

CCS Concepts

- Human-centered computing $\rightarrow$ Visualization theory, concepts and paradigms;

\section{Introduction}

Data dashboards with visualizations are routinely used across diverse domains [SCB*19], such as health care, education and assembly lines. Dashboards have been defined as visual information displays for the purpose of monitoring conditions [Few21, WSC17], but recent works suggest that they have a much broader scope [SCB*19]. We use the term dashboard to refer to a combination of multiple data visualizations and textual displays that are often interlinked/ interactive and typically arranged in a single-page layout. Most dashboards are initially created by a dashboard author and may then be used by a diverse group of users, ranging from the general public [Lau18] to analysts [GIAM16]. While analysts often have deep domain expertise, they may lack visualization literacy to interpret the data and understand the interactions with and between visualizations in a dashboard. Therefore, dashboard onboarding is required to fill the users' knowledge gaps [TLM16,CGM*17,CAS*18]. Typical methods for onboarding users to dashboards include textual descriptions, human narration, or programmed "guided tours". While there is a substantial amount of previous work on dashboard recommendation and the presentation of insights [EB12,DR04], there is a lack of research into formalizing and unifying different onboarding strategies for dashboards. We argue that a process model for dashboard onboarding can be beneficial to understanding existing techniques and facilitate the design and implementation of new onboarding approaches.
Based on the literature [CFGT21], our own experience from previous projects related to onboarding [SGP* $19, \mathrm{SWG}^{*} 21$ ], and discussions with collaboration partners from the steel and pharmaceutical industries who create and use dashboards, we know that the onboarding process can vary based on the target groups (characterized by differences in dashboard literacy). Some users may require in-depth onboarding in relation to a specific visualization type with explanation of the data sources and the model, while others require only a high-level understanding of the visualizations and how they are linked. Furthermore, onboarding techniques vary in terms of when and where they are applied. Thus, a process model for dashboard onboarding must be sufficiently flexible to correctly describe a diverse range of onboarding approaches. At the same time, it must be specific enough to help developers and researchers to better understand the building blocks of each individual approach.

The main contribution of our work is a process model for onboarding users to dashboards that fulfills these criteria. In our model, we introduce the onboarding loop alongside the dashboard usage loop (Figure 1). Inspired by the model-view-controller (MVC) software design pattern, we discuss the most important building blocks of the onboarding process. Our model gives rise to an onboarding creation pipeline in which a structured dashboard representation is combined with an onboarding narrative and a set of onboarding means (e.g., human narration, screen recording). The resulting onboarding artifacts, such as oral or video explanations, are presented to the user. We illustrate both the generalizability and specificity of our model by 


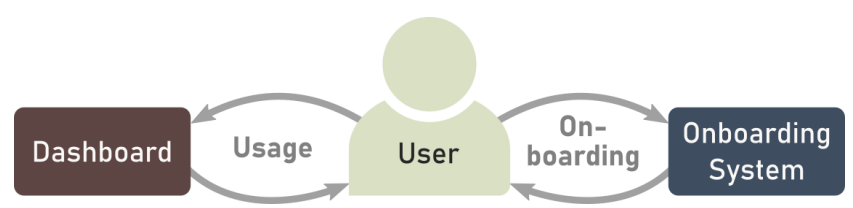

Figure 1: Introducing the dashboard onboarding loop alongside the usage loop.

showing three different onboarding approaches that can be viewed as manifestations of our model. We also demonstrate an example of onboarding performed using our process model. Additionally, based on our model, we conceptualize a hypothetical interactive and adaptive dashboard onboarding system using an AI model.

\section{Related Work}

The literature on onboarding is broad, covering multiple domains such as gaming [AOL ${ }^{*} 12$, MLS $\left.^{*} 16\right]$ and software [LGF12]. While onboarding strategies from these domains could be adapted for dashboard onboarding, we limit our discussion of related work to visualization onboarding. Note, that we focus on onboarding as opposed to guidance. The aim of guidance is to support users in performing specific tasks with visual analytic tools [CGM*17], such as orienting them during the analysis or providing suggestions about how to arrive at certain insights. In contrast, onboarding helps users to understand, interpret, and use the applied visual analytics methods, providing explanations about key features and capabilities [BMBH16, SGP*19].

For onboarding users to a single visualization, Stoiber et al. [SGP* 19] applied the five Ws and H framework to characterize the visualization onboarding space. We use a similar approach to characterize the onboarding space for dashboards in Section 3. In relation to the how, where, and when an onboarding is provided, we can further differentiate between active and passive learning approaches [TLM16]. Passive approaches for visualization onboarding include cheat sheets [WSMRB20], textual explanations [HH, Rib20], and tutorial videos [Rib20]. Sticky notes [KPS03] are textual callouts that are placed directly within dashboards. They may be passive or active, depending on whether they include a call to action. Fully active approaches onboard users while interacting with a visualization, for instance, by enabling two-way communication [EAB13, BE18], or through context-aware annotations [EB12, KPHH12]. Active approaches have been shown to be more effective than passive ones in specific cases [FDL20, KL16].

Regardless of the exact means chosen, visualization onboarding is often based on a narrative. Stoiber et al. [SWG*21], for example, separated their onboarding instructions for single visualizations into reading the chart, interacting with the chart, and using the chart. In the course of conceptual work for our process model, we realized that an onboarding narrative is particularly important for dashboards, as they include multiple visualizations and potentially complex interactions. This relates dashboard onboarding to (visual) storytelling, a common technique for communicating insights into data to users [BWF* 18]. Segel and Heer [SH10] differentiated between author-driven and user-driven stories. In the context of onboarding, we found that author-driven stories created before the onboarding process [LRIC15, BDF15] are used mainly in passive approaches. Active onboarding may also include user-driven elements, for example, when a predefined story is adapted according to the users' questions and needs. This can be handled via various storytelling techniques, such as Martini glass structure [SH10] and SlideShow [WLF*19]. Also, encoding the interaction history in the visualization guides the users to insight-driven exploration [FDPH17].

Several attempts have been made to automate the storytelling process [HDR* $\left.{ }^{*} 13, \mathrm{CPL} 20, \mathrm{SSX}^{*} 21\right]$. In Section 4.1 we introduce a structured representation of dashboards that may form the basis for automated narrative selection. This representation is closely related to the dashboard meta-models introduced by Vázquez-Ingelmo et al. [VIGPT19], augmented with a visualization grammar such as Vega-Lite [SMWH17]. While the dashboard meta-model formalizes aspects of the dashboard itself, we have noticed a lack of work on formalizing the onboarding process. We sought to fill this gap with our process model for dashboard onboarding, for which we drew inspiration from other process models related to visualization [FWR $\left.{ }^{*} 17, \mathrm{BMW} \mathrm{SGP}^{*} 19\right]$, and from the MVC software design pattern [EB11, ADK*12].

\section{Characterization of the Dashboard Onboarding Space}

To characterize the dashboard onboarding space, we adapt and refine the Ws and H questions proposed by Stoiber et al. [SGP* 19]. Their characterization focuses solely on literacy with respect to a single visualization and does not consider the interplay between the multiple different and linked visual components that form the dashboard. For the purpose of dashboard onboarding, we adjust the Ws and $\mathrm{H}$ as follows: (i) (2) WHO is the target user, (ii) $\bigcirc$ WHY is a dashboard onboarding needed, (iii) G WHAT needs to be onboarded, (iv) HOW is dashboard onboarding provided, (v) $\odot$ WHERE is dashboard onboarding provided, and (vi) $\odot$ WHEN is dashboard onboarding used. In all of these questions, we refer to both the visual and the functional genre of dashboards, as discussed by Sarikaya et al. [SCB*19].

(2) WHO needs to be onboarded is referred to as the dashboard user [TBFGC21] in our process model. Stoiber et al. [SGP*19] describe the characteristics of such a user based on their domain, data, visual encoding, interaction and analytical knowledge. While the same user traits are relevant for dashboard onboarding, we do not make them explicit in our process model as we focus on formalizing the overall process of onboarding. We also highlight the role of the onboarding author, who takes over the tasks related to the creation of the onboarding [LRIC15]. The onboarding author decides the what, how, where, and when of the onboarding process based on the overarching question of why a user needs to be onboarded.

Q WHY a dashboard requires onboarding depends on various factors, such as its data, complexity, purpose, and dashboard user characteristics. The why is a driving question behind every choice made in the onboarding phase. We consider this question to be asked before deciding on all the other Ws and H. Therefore, we couple it with the following Ws and $\mathrm{H}$ questions to highlight its significance.

GHAT can be onboarded in a dashboard includes, but is not limited to, visualizations, global filters, and text elements that provide con- 
text [VIGPT19]. The visualizations may also have specific controls that not only affect themselves but also other visualizations, through filtering or highlighting. These interactions may also have to be explained to users. To specify a dashboard's component in more detail, we further break them down into their low-level characteristics (such as marks, axis, etc.) using the Vega-lite grammar [SMWH17]. Although the list of components that can be onboarded is extensive, we use the what to refer to components that are actually explained to the user, which is a subset of all the components. The choice of why a subset is explained is made by the onboarding author depending on the purpose of onboarding, data complexity, and dashboard user characteristics, among other factors. Our interpretation of the what deviates considerably from Stoiber et al.'s definition as they use this $\mathrm{W}$ to define the onboarding space itself (i.e., what $i$ s onboarding?) [SGP*19].

HOW a dashboard onboarding is provided to the user refers to the means chosen. We use the same principles as described by Stoiber et al. [SGP*19], namely, type, context sensitivity and interaction, to describe how a dashboard onboarding is provided. For example, a human narration with a participatory dialogue serves as a means to an active onboarding approach. However, if the participatory dialogue is removed, the same narration will serve as a means to a passive onboarding approach. Other onboarding means include sticky notes, in-place annotations, narrations, video sequences, among others. Therefore, why certain means are chosen depends on the level of user engagement planned during the onboarding. Another reason can be the time and effort required to author these means, as highlighted by the interview summary by Brehmer et al. [BK22].

a dashboard onboarding is provided can vary between being integrated directly into a dashboard or being presented in an external source [SGP* 19]. When embedded directly in the dashboard, it can be displayed as overlay information by means of, for example, sticky notes that explain the visual components [KPS03] or a stepby-step guide positioned in a side panel next to the dashboard and can be opened on demand [Ber04]. In contrast, external sources are accessible to the user at any time and are independent of the direct use of the dashboard. This, for example, includes screen recordings and textual documentations. The interviews by Brehmer et al. [BK22] indicate that onboarding authors consider aspects like the type and purpose of content consumption to assess why a specific placement may be favorable.

() WHEN a dashboard onboarding is provided depends on whether the user needs to be onboarded before or during interaction with a dashboard (same as in [SGP* 19]). Textual onboarding or video tutorials can, for instance, be presented before interacting with a dashboard. In the case of an integrated dashboard onboarding, the onboarding artifacts are shown to the user while interacting with a dashboard. Why an onboarding author chooses a synchronous versus an asynchronous strategy may depend on the possibility of in-person meetings with the users or whether onboarding material should be available for later usage [BK22].

\section{Process Model}

Dashboard onboarding is a multifaceted problem. While several of its aspects have been touched upon in related work, we found a lack of a solid "scaffold" to support, sustain, and consolidate the discussion of dashboard onboarding in the visualization community. We designed our process model to provide such a scaffold. In order to inspire future work on onboarding authoring, implementation, and evaluation over a wide range of different onboarding strategies, our design process for the model was guided by three requirements. We wanted the model to be:

- general enough to cover all existing onboarding strategies without having to introduce new concepts for each particular case;

- specific enough to allow detailed description of individual strategies, for instance, by mapping particular elements of existing strategies to general concepts; and

- actionable, which means that adhering to it should facilitate the design of new onboarding strategies.

While these requirements may seem obvious, it was not clear how to arrive at a model that would meet all of them simultaneously. We started the design process for the model with an analysis of existing dashboard onboarding strategies. By extracting commonalities and differences of various strategies, and based on related work, we identified fundamental steps and building blocks in the creation and application of the onboarding that we wanted to be reflected in the model. We then iteratively created models and checked whether they met the requirements.

We created several "bottom-up" models based on specific strategies, however, the models generalized poorly. We further created "top-down" models from broad principles but found that in certain cases they require too many modifications, or are too abstract to be actionable. In early versions of our model, we struggled with how to reflect various user roles (see discussion in Section 7.2). Sketches for discarded models can be found in the supplementary material [sup21].

After many iterations, we finally converged at a process model that met all requirements to our satisfaction. This model introduces the onboarding loop as an equally important counterpart to the dashboard usage loop (see Figure 1). We based the onboarding loop's internal structures on the MVC software design pattern [ADK*12]. Our choice to model the onboarding loop in a similar way was motivated not only by aesthetic considerations, but also by the actionability requirement.

In the following sections, we first look at the usage loop in more detail and explain how it leads to a structured representation of a dashboard with its components (Section 4.1). We then unpack the onboarding loop, discuss its structural similarity to the usage loop, and derive a "recipe" that describes which parts need to be specified when creating an onboarding system/strategy (Section 4.2).

\subsection{Dashboard Usage Loop}

Dashboards can be described as a collection of typically linked visualizations of a dataset that are controlled by a user through a set of interactions. To formalize this description, we make use of the MVC software design pattern [ADK*12], which allows us to break the dashboard down into three conceptual building blocks (see left part of Figure 2): (i) the underlying data model, (ii) the dashboard's visual components, and (iii) the control elements or interactions. Typically, dashboards do not reveal the underlying 


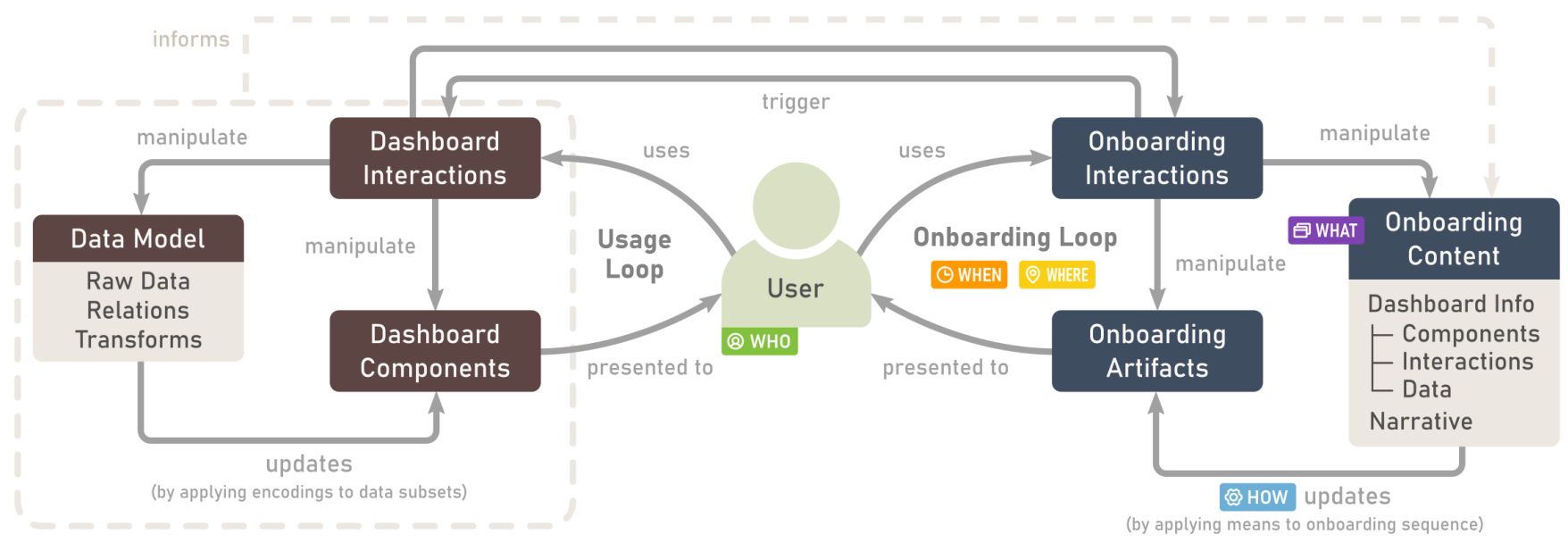

Figure 2: Process model for onboarding users to dashboards. In the usage loop, the user manipulates the underlying data model and/or the visual dashboard components through interactions. In the onboarding loop, the user likewise manipulates onboarding artifacts and/or the underlying onboarding content through interactions. The onboarding content is informed by a structured representation of the inner workings of the dashboard.

data model and logic to the end users, but let them manipulate the data model indirectly through interactions. Such an indirect manipulation of the data model frequently results in an update of the visual components presented to the users. Consequently, it is also possible that interactions directly affect the visual components without altering the data model. We explain the roles of the three building blocks in the onboarding loop in more detail, making use of a simplified version of the New Hires dashboard provided by Microsoft Power BI [hum21], shown in Figure 3.

The Data Model broadly consists of the raw data, relations between data subsets, and the transformations applied to the raw data [PC05]. The data model outputs transformed subsets of the data that can later be presented by visual components. For the given example dashboard (Figure 3), the data consists of business unit, region, ethnicity, and time.

The Dashboard Components are all visual components that are presented to the user. In particular, this includes visualizations such as bar and line charts. Most dashboard components are constructed by applying specific encodings to data subsets which are output by the data model. Details about these components can be listed with the aid of (i) the dashboard meta model presented by Ingelmo et. al [VIGPT19] combined with (ii) a visualization grammar, such as Vega-Lite [SMWH17]. The meta-model for dashboards first enlists all the components required to create a dashboard. Combining the information with the Vega-Lite grammar breaks down individual components into low-level concepts such as axes, marks, and channels. In our guiding example, the main visual components are two bar charts, a line chart, and the key performance indicator (KPI) at the top left (see schematic in Figure 3 1).

The Dashboard Interactions are all interactive components in the dashboard that can be controlled by a user through input devices (e.g., mouse and/or keyboard). In typical dashboards, interactions are closely tied to visual components to create an immersive dashboard experience [MM21]. This close relationship has led to ambiguities concerning the view and controller parts of the MVC pattern [ADK*12]. Some interactions, such as filtering or selecting, manipulate the data model. Other interactions, such as zooming, panning, or highlighting, may directly influence the visual components without altering the data model. For instance, filtering through check boxes is one of the main interactions technique used in our guiding example.

The three building blocks of the dashboard usage loop lend themselves well to a structured representation of a dashboard that can be later used in the onboarding process. The first step is to create a list with all components. For each component, we note the data subset required for its creation and list all its characteristics (e.g., mark type and channels). Figure 32 shows such a representation for the bar chart in our guiding example.

In the next step, we list interactions tied to the visual components. For linked and coordinated views, interactions result in edges between components. Thus, we arrive at a component graph that reflects the relationships between the dashboard's visual components. In our example, selection of a bar in the respective chart triggers filtering of the data used in both the line chart and the KPI. The selection also leads to highlighting in the stacked bar chart. In the component graph, we represent this information as directed edges from the node of the bar chart to the nodes of the other components (see simplified example of filtering components in Figure 32 ).

To make the component graph useful for the onboarding process, it needs to be enriched with explanations. The explanations can be descriptions of the purpose of the dashboard, information about specific tasks addressed by the components [AES05], or exemplary insights that can be gained by using them. Explanations can also include information about the low-level characteristics of a component, for instance, why a certain mark type or color scheme is used. Such explanations can be provided by the dashboard/onboarding 


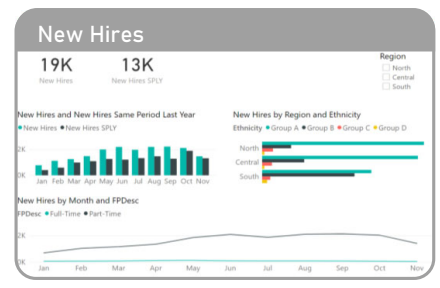

Schematic Respresentation of the Dashboard
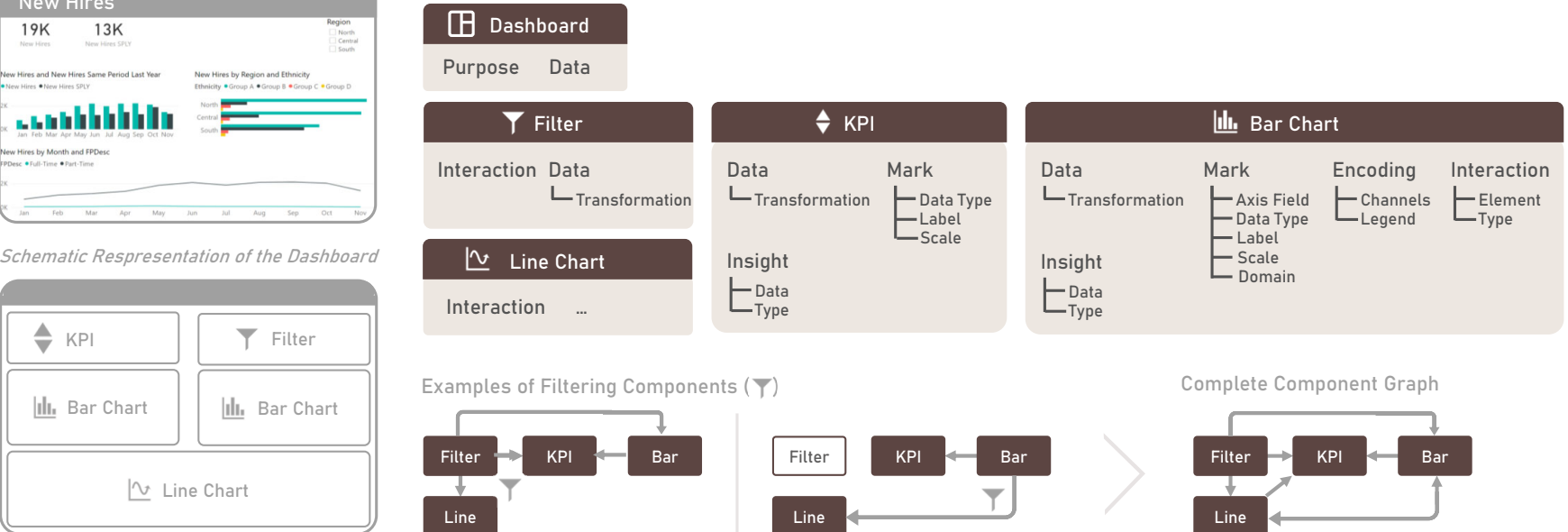

-Type
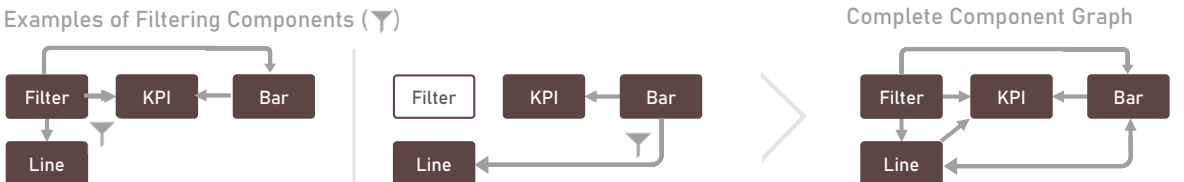

3 Depth-First Narrative
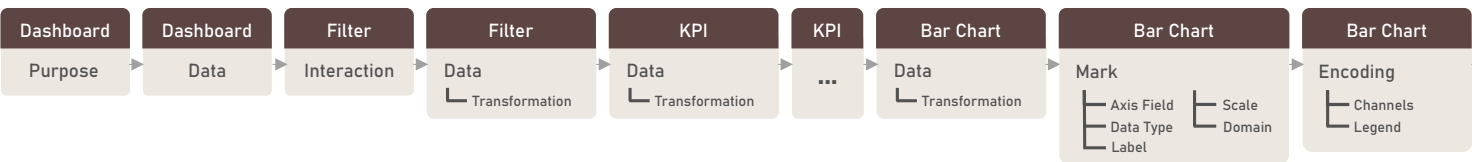

Figure 3: 1 Example dashboard from Microsoft Power BI using six visual components: two KPIs, one filter component, two bar charts, and one line chart. 2 List of all visual components, and simplified representations of interactions through filtering using either the filter or the bar chart component. The complete component graph shows the inter-dependencies between all four components. A fully fledged component graph with relationships at the lowest component level can be retrieved from the supplementary material [sup21]. 3 Traversal strategy based on a depth-first narrative to explain all dashboard components one by one.

author or inferred automatically. In our sample dashboard, one example of an insight is that the grouped bar chart shows the highest number of new hires in the region North and Group A.

Note that the enriched component graph is not always constructed explicitly. In many cases, the onboarding author has only a mental model of the dashboard's components, interactions, and insights that they want to explain to the user. Regardless of whether the structured representation of the dashboard is made explicit as an enriched component graph or whether the onboarding author internalized it as a mental model, this representation forms the basis of the onboarding content in the onboarding loop (dashed arrow in Figure 2).

\subsection{Onboarding Loop}

The onboarding loop makes use of the information contained in the structured representation explained above and, unpacking it describes how this information is processed to finally present it to the user. Similar to the dashboard usage loop, the onboarding loop comprises three building blocks: (i) the onboarding content, (ii) the onboarding artifacts, and (iii) the onboarding interactions.

The Onboarding Content consists of the structured dashboard representation together with an onboarding narrative. This narrative drives the traversal of the component graph for the purpose of storytelling, and results in a sequence of components and/or inter- actions, enriched with their explanations (目 WHAT). The narrative is specified directly by the onboarding author or determined by an algorithm. Examples of simple traversal strategies are depth-first traversal (give all or most details for each component immediately) and layout-based strategies (explaining dashboard components in the same order as they appear in the dashboard, e.g., from left to right or top to bottom). More insight-focused narratives may lead to more complex traversals and usually have to be hand-crafted by the onboarding author. A simple depth-first traversal for our guiding example is shown in Figure $3 \mathbf{3}$.

In the usage loop, carefully selected encodings are applied to data subsets that are output by the data model to construct the visual dashboard components. In a conceptually similar way, carefully selected onboarding means ( $\mathrm{HOW}$ ) are applied to the sequences derived from the narrative, which result in Onboarding Artifacts. As outlined in Section 3, onboarding means can range from (human) narrations to tooltip information and sticky notes [EB12]. Depending on the means, the artifacts can be visual, auditory, or textual, for instance.

The onboarding artifacts can be either embedded directly within the dashboard or presented to the user elsewhere. (๑) WHERE the artifacts are presented depends largely on the means chosen (㲅 HOW ). For example, in the case of video onboarding, the onboarding inter- 


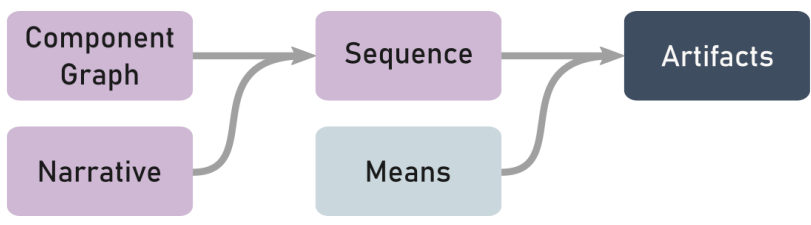

Figure 4: "Recipe" for constructing onboarding artifacts. The component graph is traversed based on a narrative, resulting in an ordered sequence to which onboarding means are applied.

face is a video player and the means of onboarding may be a screen recording of the dashboard along with human narration.

Furthermore, the presenter can decide $\odot$ WHEN to onboard the user: either before or while interacting with the dashboard. In the case of a programmed guided tour embedded within the dashboard, the onboarding can be retrieved on demand during the dashboard usage; see example in Section 5.3.

The Onboarding Interactions provide the user with functionality to control the onboarding process. Depending on the type of onboarding, the interactions manipulate different aspects. In static onboarding, interactions merely affect the onboarding artifacts. For instance, in a video tutorial, the script has already been written and the screen has been recorded prior to the onboarding. Only limited controls are given to the user, such as pausing and continuing a video or going back to a specific timestamp/sequence (see example in Section 5.1). In an adaptive onboarding, the interactions trigger updates of the underlying onboarding content. This means that a human presenter may come up with a new onboarding narrative based on a question asked by the user (see example in Section 5.4). In the exceptional case in which interface and dashboard interactions are directly connected, a user input in the onboarding interface may trigger a change within the dashboard. Conversely, an active onboarding system may listen to events in the dashboard, which may result in updated onboarding artifacts or an updated narrative.

Each block and the connections between them in the general onboarding process model shown in Figure 2 depends on the specific use case.

\section{Manifestation of the Process Model in Various Onboarding Scenarios}

In this section, we show how real-world onboarding scenarios can be described using our process model. We present four different onboarding strategies - a video tutorial, a static textual documentation, a programmed guided tour, and an interactive onboarding session with a human narrator-and discuss the role of each part of the process model in each case. In the first three examples, we analyzed existing techniques and mapped them to our process model. In these cases, we also hypothesize that the component graph represents the mental model of the onboarding author. The fourth example (Section 5.4) describes an onboarding based on our process model, which we carried out with a collaborator. Finally, we describe a hypothetical AI-powered adaptive onboarding system based on our process model. The detailed onboarding process for these examples can be found in the supplementary material [sup21].

\subsection{Onboarding Video}

Video tutorials allow the onboarding author to prepare a prescribed story that enables any user to play and recall it sequence by sequence. We screened multiple video tutorials (e.g., on YouTube) that introduce interactive dashboards created for visual analytics tools and chose one that required little domain knowledge. Below we describe a video tutorial using a sales dashboard developed in Microsoft Power BI [Tra21] and show the corresponding process model in Figure 5.

Before recording the tutorial, we assume that the onboarding author chose a narrative based on an example usage scenario of the dashboard. To come up with this narrative, the onboarding author assumed the role of the dashboard user and completed several iterations of the dashboard usage loop. This example usage informed a narrative that covers dashboard overview, filter selection, bar chart explanation with drill-down, and KPIs information (Đ WHAT). In the case of the video tutorial, the onboarding author decided to record the screen and use an oral narration as the onboarding means ( $\mathrm{HOW}$ ). The resulting onboarding artifact is the video, which is presented to the user on the video platform YouTube ( $\odot$ WHERE).

As shown in Figure 5, during the onboarding, the user has the ability to navigate through the video using typical controls such as pausing, fast-forwarding, or jumping to specific sections. While these interactions affect the state of the onboarding artifact that is being shown to the user (i.e., the current video frame), there is no way of triggering an update of the onboarding content. In this sense, onboarding using a typical video tutorial is static, which is reflected by the greyed-out edge between the onboarding interactions and the onboarding content in Figure 5.

The users can choose whether they want to watch the video prior to using the dashboard or whether they want to try to recreate the presented interactions in the dashboard, for instance, while viewing the video ( $\odot$ WHEN ) on a second screen. There is no explicit connection between the onboarding and the usage loops, which is indicated by greyed-out edges between the user and the dashboard usage loop (in Figure 5).

\subsection{Textual Explanation}

For the second example, we used the regional information dashboard about climate change projections from the IPCC WGI Interactive

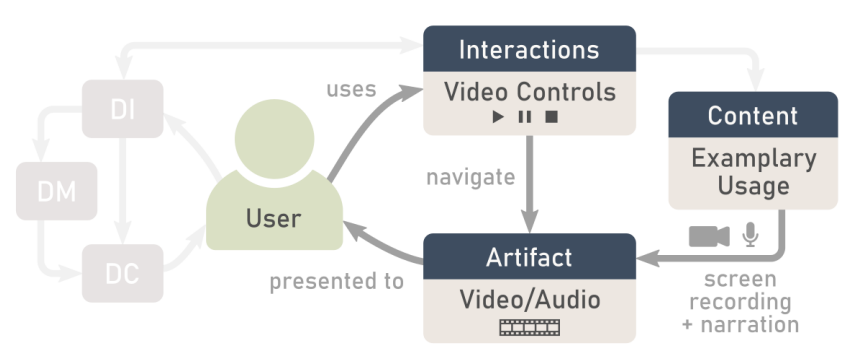

Figure 5: Onboarding with a video tutorial, described using our process model. 


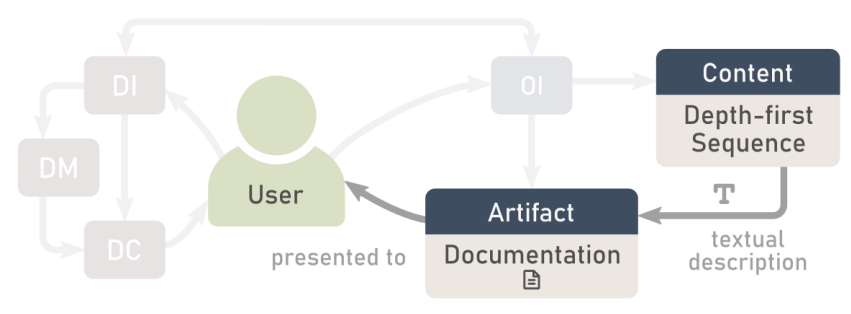

Figure 6: Onboarding with a text document, described using our process model.

Atlas [at121b, at121a]. Here, the onboarding author enabled two different ways of user onboarding: textual documentation and a programmed guided tour embedded in the dashboard. This section focuses on the documentation, and the next one (Section 5.3) on the guided tour.

The textual documentation includes information about the dashboard components and their corresponding interactions. For each component, the document describes which data subset is visualized, which encodings are applied, and which interactions are available ( HAT ). Based on this description, the traversal of the component graph can be considered depth-first.

The onboarding author decided to use a textual format as onboarding means ( HOW ) and the documentation as the resulting onboarding artifact, which can be accessed via the web ( $\odot$ WHERE ) [at121b] (see Figure 6).

Although the document includes hyperlinks to external resources, we do not consider them to be controls. Instead, we assume that the brief documentation is read from top to bottom and that clicking on external links is equivalent to stopping the onboarding. Without proper onboarding controls, the process model (Figure 6) no longer includes an onboarding loop. Instead, the one-time consumption of the external source, in this case a static document, is reflected in the fact that the user has no connection to any other location during onboarding. The document can be accessed by the user at any time before, during, or after the dashboard usage (C WHEN ). As in the video case, there is no explicit connection between usage and onboarding in this case.

\subsection{Programmed Guided Tour}

As introduced in the previous section, the programmed guided tour example uses the same data as in the textual explanation. Compared to the textual explanation, it provides slightly less details about the components for the onboarding.

In this case, we infer that the program follows a breadth-first traversal of each component, as the sequence with its required components (는 WHAT is fixed and programmed in a way that cannot be adapted. Onboarding is represented using the means of highlighting and callouts or tooltips ( $\mathrm{HOW}$ ), which are overlaid on the dashboard itself ( $\odot$ WHERE). The programmed guided tour is presented as a simple stepper interface when accessing the dashboard for the first time (@ WHEN ). It can be accessed at any time when interacting with the dashboard by clicking on the information icon.
The stepper interface lets the user navigate the sequence of explanations, allowing them to either back to the previous step, proceed to the next one, or to end the onboarding process. This predefined onboarding narrative and the resulting sequence cannot be customized by the user. It is reflected by a grayed-out edge between the onboarding interactions and the content in the process model (Figure 7). During the onboarding process, the usage of the dashboard is either blocked or limited and has no effect on the onboarding narrative. For the most part, this leads to disconnected usage and onboarding loops. In only one case does the interface explicitly encourage the user to interact with the dashboard by selecting a region in the map to continue the onboarding process. This one-time interaction is indicated in the process model (Figure 7) by the edge from user to select region in the dashboard. This selection triggers the onboarding stepper interface once and the onboarding process continues. The subsequent onboarding steps are again controlled through the overlaid stepper interface.

Upon restarting the onboarding process during a dashboard usage, the user has to start the predefined onboarding story again from the first component, regardless of the already introduced components. The system does not save any past interactions with the dashboard or the onboarding, and the traversal order of the component graph is fully fixed.

\subsection{Interactive Onboarding with Human Presenter}

For this example, we authored an onboarding for an interactive dashboard based on our process model. We presented it to a dashboard user in the course of a collaboration with the steel industry.

In the previous examples, onboarding was designed for heterogeneous groups of users. In this case, we present an onboarding that was tailored to specific users who had a comprehensive data and domain knowledge. They were not new to interactive dashboards overall, however, a beginner in the field of visualization. For this purpose, we created a list of components which required onboarding (曰 WHAT ) from our experience. We then listed two possible predefined sequences for explanation and chose the one which increased the complexity of onboarding gradually. The onboarding took place virtually using screen sharing via an internal communication platform for teleconferences ( $\odot$ WHERE). The narrative was provided to the user in the form of oral explanations ( HOW ). As we made use of visualizations in which the user had little to no prior experience, we provided the onboarding before the first-time use of the dashboard (Q WHEN). This scenario is reflected in the process model (Figure 8) by the lack of a "use" connection from the user to the dashboard, while the "presented to" connection from the dashboard components to the user still exists.

After our explanation, the user posed questions to us, which is represented by the Interactions block in Figure 8 . They also used the shared screen to annotate the visuals for clarifying the explanations provided by us. The user's questions either (i) prompted a change in the oral explanation, which led us to rephrase what was said without changing the onboarding strategy; (ii) caused us to update the underlying onboarding model, for example, by changing the narrative to explain components of interest; or (iii) triggered an interaction in the dashboard, potentially requiring an update to the narrative to reflect the resulting changes. 
After one hour of onboarding, the user was done posing questions on various visualization types and interactions. At this point the user had gained an understanding of how to derive actionable insights from the dashboard, indicating a successful session. We discuss the extent to which the process model helped us in the authoring and evaluation of our onboarding in Section 6.

\subsection{AI-based Onboarding}

Finally, we applied our process model to a hypothetical onboarding scenario, which tries to overcome existing flaws of human-narrated onboarding scenarios described in Section 5.4. One of its obvious flaws is that, once the onboarding has been completed, it cannot be repeated or accessed on demand. Further questions can be asked by sending requests to the onboarding author, but the answers may not be provided immediately. Thus, we imagine an AI-based solution to this problem that can be represented with our process model. In contrast to the human narration, such an AI-powered onboarding can be accessed by the user flexibly at any time.

In our proposed automated onboarding solution, the user can initiate the onboarding process by typing in a query such as "Onboard me to the dashboard". This triggers an AI model to derive a component graph from the dashboard with some example insights. With the help of this component graph and a predefined, initial traversal strategy (e.g., depth-first), the AI model can create an onboarding narrative. This narrative may be presented using callouts or tooltips ( HOW ), overlaid directly onto the relevant dashboard components ( $\odot$ WHERE ). The user can then choose to follow the predefined narrative, skip steps, or stop it by interacting with the callouts and/or the dashboard. When the user poses a question to the interface, an AI model could respond in ways that resemble a human reaction by (i) rephrasing the current explanation, (ii) changing the narrative, or (iii) initiating changes directly in the dashboard. Possible strategies for updating the narrative could be based on the user's previous interactions with the dashboard and/or the onboarding interface. For parsing the questions and relating them to individual dashboard components, we envision a natural language processing model similar to LILY [QDRH19], which was developed for creating dashboards from written prompts.

The process model for such an AI-based onboarding looks like to the one for an interactive session with a human presenter (Figure 8), except for two additional edges. First, since such an onboarding would take place synchronously with the dashboard usage, the edge from the user to the dashboard is present. Second, as the AI model can update the onboarding based on interactions between the user and the dashboard, the second, left-to-right "trigger" edge between the interactions would be present. As a result, this hypothetical AIpowered onboarding process would make use of all connections in the general model shown in Figure 2.

\section{Using the Process Model}

In a data-driven world, use of dashboards is inevitable. As highlighted by Sarikaya et. al [SCB* 19] and Tory et. al [TBFGC21], it is important to support the end users in dashboard literacy. Answering their call to action, we provide practical advice to those who want to support users in understanding dashboards. Accordingly, in this section, we discuss ideas and insights gained from onboarding in Section 5.4 by applying our process model for dashboard onboarding in practice. We group these ideas into three phases that reflect the onboarding process: authoring, implementation, and evaluation.

\subsection{Onboarding Authoring}

The authoring phase of a dashboard requires the author to choose subsets of data to be visualized and presented to the user. Similarly, the authoring phase of the onboarding requires the author to select specific visual components and interactions that should be explained to the user.

Previous approaches aimed to assist the author in the dashboard authoring phase by selecting the needed data subsets and/or visualizations [THHS16]. However, there is insufficient literature on how to author the onboarding phase. In this section, we describe how our contribution can potentially facilitate various aspects of the onboarding authoring.

The structured representation, introduced in Section 4.1, can be made explicit by the onboarding authors to identify dashboard components, interactions, or pieces of data-related information that may be vital to the end user. For the onboarding case in Section 5.4, we used the structured representation to gain a first idea of narration sequences that arose "naturally" from the dashboard structure. It indicated that the time slicer had the most relations to the other components. This helped us in deciding two possible narrative sequences, where the slicer could be introduced first or towards the

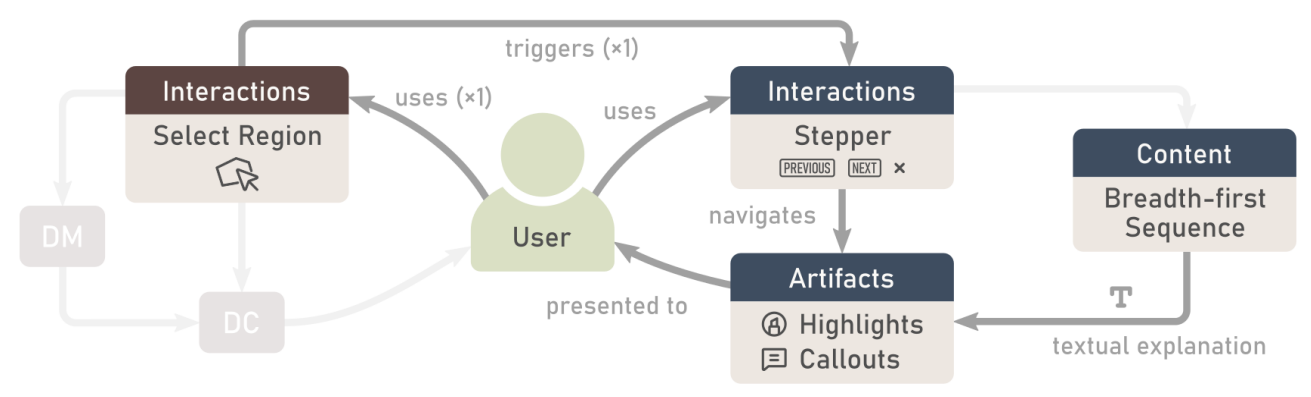

Figure 7: Onboarding with a programmed "guided tour", described using our process model. 


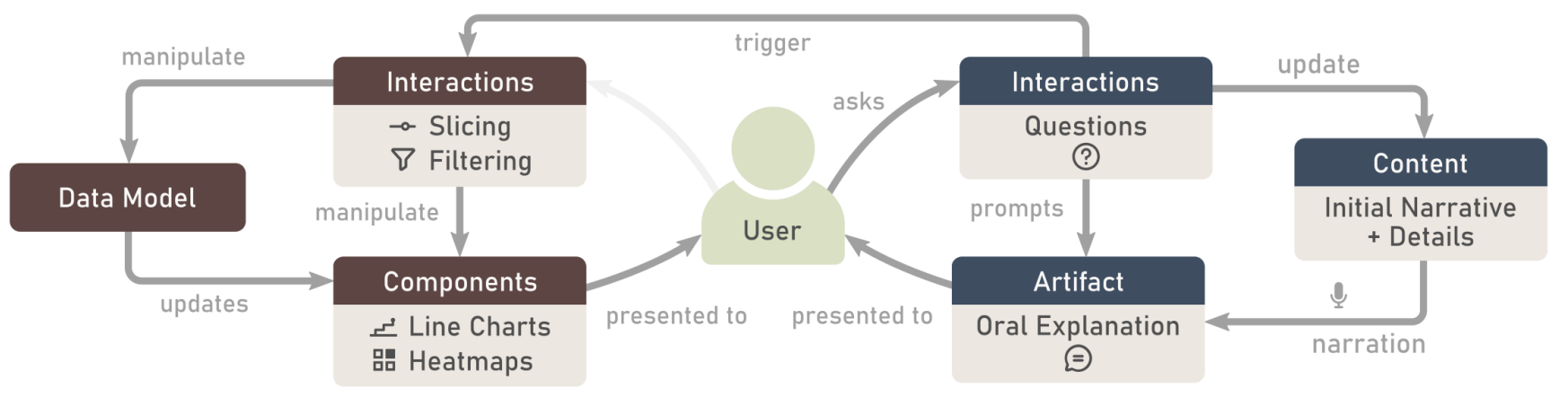

Figure 8: Onboarding in an interactive session with a human presenter, described using our process model.

end. We decided to go with the latter to gradually increase the complexity of onboarding. Additionally, attaching small explanations to individual nodes in the component graph allows the author to break down a potentially complex onboarding narrative into manageable parts. This may also enable the author to reuse individual explanations, regardless of the specific narrative chosen or onboarding means applied later in the process.

Finally, the onboarding recipe, shown in Figure 4, describes how a specific set of onboarding artifacts can result from choices made during the authoring phase. Even though the choices of narrative and means may be interdependent and strongly coupled to the overarching question of $Q$ WHY an onboarding is needed, the recipe reveals that one onboarding strategy may be transformed into another by choosing alternative means. For instance, using the onboarding recipe, it should be straightforward to create a programmed guided tour based on a textual documentation.

\subsection{Onboarding Implementation}

The structure we chose for both the usage and the onboarding loops, is closely based on the MVC software design pattern. Thus, our process model can serve as a guide in the implementation of new onboarding systems. For example, it can be used to infer when the dashboard needs to send information to the onboarding interface or vice versa. More importantly, the process model can help onboarding authors to identify when an update of the underlying narrative is necessary and when updating only the onboarding artifacts is sufficient (e.g., choosing a different explanation strategy for the same visual component).

Since the structured representation is the foundation of the onboarding loop, we present our thoughts on how to automatically extract this representation. The first step is to extract the components of the dashboard and their relationships. For an embedded Microsoft Power BI dashboard [emb21], Embedded Report APIs, such as Power BI Client API [pow21], can be used to this end. We assume that similar workflows are viable for other tools such as Tableau [tab21]. In second step, the enrichment of the structured representation with explanations must be formalized. This can easily be specified through an approach similar to Encodable [Won20], a configurable grammar for visualization. Further thoughts on how to make use of Encodable can be found in the supplementary material [sup21]. After creation of the fully enriched graph, any graph traversal strategy can be implemented as a default strategy for the onboarding. The resulting sequence can then be shown as a textual description using callouts, overlaid directly on the dashboard for the user. In the case of an adaptive onboarding, the onboarding controls can allow the user to skip or access parts of onboarding based on their needs. Note that this is only one way of implementing the process model, and the capabilities of the implemented model can be enhanced in various ways, for instance, by inferring user preferences.

\subsection{Onboarding Evaluation}

Section 5 lists several examples of real-world onboarding processes described with our process model. We believe that taking existing onboarding sessions and phrasing them in terms of the process model can help presenters of dashboards to identify pain points, either regarding the dashboard usage or the quality of the onboarding. In case of Section 5.4, we recorded the session to analyze when it would have been more appropriate to show something directly in the dashboard or when it would have been more effective to update the narrative based on the user's needs. This helped us to improve our narrative for the next sessions, for instance, by adding the explanation of the line chart again after the interaction with the time slicer.

\section{Discussion}

In this section, we describe the extent to which our process model can be used for informing and evaluating onboarding techniques and how our model can, in turn, be evaluated by such a technique. We also discuss future strategies for evaluating the process model. Finally, we share the lessons learned by highlighting some of these techniques during the creation of our process model.

\subsection{Evaluation}

As described in Section 6.1, our model can only inform the authoring and evaluation process to the extent of providing a scaffold for onboarding a user. It is up to the onboarding author to make the final choices required for onboarding, weighing additional factors which 
are beyond the scope of this process model. Some of these factors are the author's presentation, engagement and language skills, the amount of user interaction planned, the skill set of the user, which all have a high impact on the onboarding process.

Failing to consider these factors and making incorrect choices for the questions raised by our model, can lead to an ineffective onboarding. Therefore, evaluating a specific onboarding-even if it was created based on our model—does not directly evaluate the model but rather the multitude of choices made by the onboarding author along the way.

One way to evaluate the process model is to test it against new onboarding strategies (as done in Section 5) to determine where the process model fails to describe them. Although we covered diverse cases in Section 5, we acknowledge that the proof of generalizability is limited and can only be solidified by future applications of the model. Therefore, as a next step, we plan to inform and evaluate future onboarding with our collaborators using our process model to establish its validity.

\subsection{Lessons Learned}

We share the lessons learned during the course of our work. We briefly discussed the main reason behind discarding most of the initial versions of our process model in Section 4. Here, we describe additional complications arising from the interplay among different user roles as indicated in Figure 9. Even without unpacking the usage and the onboarding loops, making all roles explicit resulted in a highly complex structure.

In order to reduce the complexity of the model, we decided to remove the dashboard creation loop as it is always asynchronous with the usage and onboarding loops. After all, the dashboard has already been created at the time of onboarding.

Based on this decision, we reconsidered the onboarding authoring

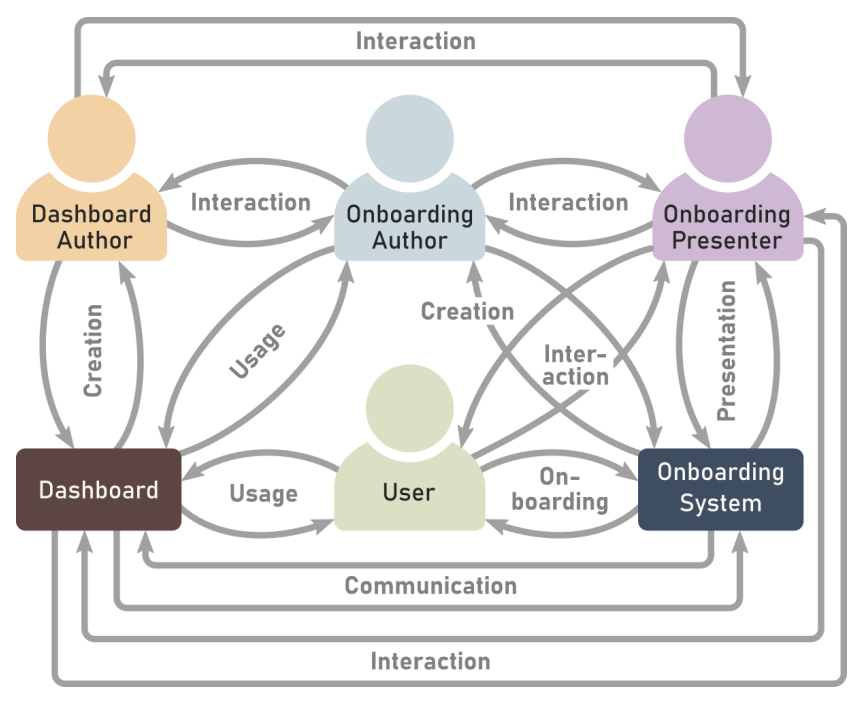

Figure 9: Initial process model showing four roles involved in the dashboard onboarding pipeline. process. Here, we realized that the onboarding authoring could either be done once, like in the case of tutorial videos or textual documentation, or repeatedly, like in the case of an interactive onboarding with a human presenter. In the latter, the presenter may need to come up with a new narrative on the fly, temporarily assuming the role of the author. Thus, we faced two challenges. One, we could no longer clearly distinguish between the onboarding author and the onboarding presenter. Two, our model had to allow dynamic updates of the onboarding content. We addressed both of these challenges by making all roles (apart from the user) implicit in the model. Rather than focusing on who updates the onboarding content, we focused on the actions that trigger updates.

After multiple iterations of process models, we also realized two additional advantages of this viewpoint. First, it allowed us to draw parallels between the interactively updated views of the dashboard with the dynamically updated content of the onboarding, leading to a symmetric process model. Second, it naturally led to the user being placed at the center of the usage and onboarding process as the sole explicit role.

\section{Conclusion}

Supporting users in their dashboard literacy is a major challenge [SCB*19]. We have presented a process model for dashboard onboarding which formalizes and unifies various onboarding strategies. We therefore introduced the usage loop, where users interact with the dashboard which results in the manipulation of either the underlying data model or the dashboard components. Alongside the dashboard usage loop, we introduced the onboarding loop, where the user manipulates onboarding artifacts or the underlying content through interactions. We demonstrate the generalizability of our model by applying it to four real-world examples (video, textual explanation, programmed guided tour, and interactive onboarding with human presenter) and proposed a hypothetical usage scenario using an AI-based onboarding which sought to solve the flaw in interactive onboarding with a human presenter. Furthermore, we provided actionable advice for developing new dashboard onboarding systems systematically and efficiently. We are confident that our process model will help to create a structured dashboard onboarding and to improve user understanding of dashboards.

\section{Acknowledgements}

This work was supported in part by the FFG (881844). Pro ${ }^{2}$ Future is funded within the Austrian COMET (Competence Centers for Excellent Technologies) Program under the auspices of the Austrian Federal Ministry for Climate Action, Environment, Energy, Mobility, Innovation and Technology, the Austrian Federal Ministry for Digital and Economic Affairs and of the States of Upper Austria and Styria. COMET is managed by the Austrian Research Promotion Agency FFG. Additional support was granted by the State of Upper Austria and the Austrian Federal Ministry of Education, Science and Research via the LIT - Linz Institute of Technology (LIT-2019-7SEE-117), and by the State of Upper Austria (Human-Interpretable Machine Learning). This paper was written in collaboration with the VRVis Competence Center. VRVis is funded by BMVIT, BMWFW, Styria, SFG and Vienna Business Agency in the scope of COMET (854174). 


\section{References}

[ADK*12] ANDREEVA J., DZHunov I., KARAVAKIS E., Kokoszkiewicz L., Nowotka M., Saiz P., Tuckett D.: Designing and developing portable large-scale JavaScript web applications within the Experiment Dashboard framework. Journal of Physics: Conference Series 396, 5 (2012), 052069. doi : 10.1088/1742-6596/396/5/052069. $2,3,4$

[AES05] AmAR R., EAGAN J., STASKo J.: Low-level components of analytic activity in information visualization. In Proceedings of the IEEE Symposium on Information Visualization (InfoVis '05) (2005), IEEE. doi:10.1109/INFVIS.2005.1532136. 4

[AOL*12] ANDERSEN E., O'Rourke E., LIU Y.-E., SNIDER R., LOWDermilk J., Truong D., CoOper S., Popovic Z.: The impact of tutorials on games of varying complexity. In Proceedings of the ACM annual conference on Human Factors in Computing Systems (2012), ACM Press, p. 59. doi : 10.1145/2207676.2207687. 2

[at121a] Atlas data sources, 2021. Accessed: 2021-11-22. URL: https : //github.com/IPCC-WG1/Atlas/tree/main/data-sources. 7

[at121b] Ipcc wgi interactive atlas: Regional information, 2021. Accessed: 2021-11-22. URL: https://interactive-atlas.ipcc.ch/ regional-information. 7

[BDF15] Boy J., Detienne F., Fekete J.-D.: Storytelling in Information Visualizations: Does It Engage Users to Explore Data? In Proceedings of the SIGCHI Conference on Human Factors in Computing Systems (2015), ACM, pp. 1449-1458. doi:10.1145/2702123.2702452. 2

[BE18] Batch A., ELmQvist N.: The Interactive Visualization Gap in Initial Exploratory Data Analysis. IEEE Transactions on Visualization and Computer Graphics 24, 1 (2018), 278-287. doi:10.1109/TVCG. 2017.2743990. 2

[Ber04] Berkeley C. P. P.: Adobe photoshop cs, 2004. Accessed 2021-11-22. 3

[BK22] Brehmer M., Kosara R.: From Jam Session to Recital: Synchronous Communication and Collaboration Around Data in Organizations. IEEE Transactions on Visualization and Computer Graphics 28, (2022), 1139-1149. doi:10.1109/TVCG.2021.3114760. 3

[BMBH16] BöRner K., MALtese A., BALliet R. N., Heimlich J.: Investigating Aspects of Data Visualization Literacy Using 20 Information Visualizations and 273 Science Museum Visitors. Information Visualization 15, 3 (2016), 198-213. doi : 10.1177/1473871615594652. 2

[BMW] BRYAN C., MA K.-L., WoOdRING J.: Temporal Summary Images: An Approach to Narrative Visualization via Interactive Annotation Generation and Placement. IEEE Transactions on Visualization and Computer Graphics 23, 1. doi : 10.1109/TVCG. 2016.2598876. 2

[BWF*18] Bach B., Wang Z., Farinella M., Murray-Rust D., HENRY Riche N.: Design Patterns for Data Comics. In Proceedings of the CHI Conference on Human Factors in Computing Systems (2018), ACM, pp. 38:1-38:12. doi : 10.1145/3173574.3173612. 2

[CAS*18] Collins C., ANDrienko N., SChreck T., YANG J., Choo J., ENGELKE U., JeNA A., DWYER T: Guidance in the human-machine analytics process. Visual Informatics 2, 3 (2018), 166-180. doi:10. 1016/j.visinf.2018.09.003.

[CFGT21] Crisan A., Fiore-Gartland B., Tory M.: Passing the Data Baton : A Retrospective Analysis on Data Science Work and Workers. IEEE Transactions on Visualization and Computer Graphics 27, 2 (2021), 1860-1870. doi:10.1109/TVCG. 2020.3030340. 1

[CGM*17] Ceneda D., Gschwandtner T., May T., Miksch S., SChUlZ H.-J., STREIT M., TOMINSKI C.: Characterizing Guidance in Visual Analytics. IEEE Transactions on Visualization and Computer Graphics (VAST'16) 23, 1 (2017), 111-120. doi:10.1109/TVCG. 2016 2598468. 1,2

[CPL20] CAO Y.-R., PAN J.-Y., LIN W.-C.: User-oriented Generation of Contextual Visualization Sequences. In Extended Abstracts of the CHI Conference on Human Factors in Computing Systems (2020), ACM pp. 1-8. doi: $10.1145 / 3334480.3383057 .2$
[DR04] DwORMAN G., RoSEnBaUm S.: Helping users to use help: improving interaction with help systems. In Extended abstracts of the conference on Human factors and computing systems (2004), ACM Press, p. 1717. doi : 10.1145/985921.986198. 1

[EAB13] Elias M., Aufaure M.-A., Bezerianos A.: Storytelling in Visual Analytics Tools for Business Intelligence. In Human-Computer Interaction - INTERACT 2013, vol. 8119. Springer Berlin Heidelberg, 2013, pp. 280-297. doi:10.1007/978-3-642-40477-1_18. 2

[EB11] Elias M., Bezerianos A.: Exploration Views: Understanding Dashboard Creation and Customization for Visualization Novices. In Human-Computer Interaction - INTERACT '11, vol. 6949 Springer Berlin Heidelberg, 2011, pp. 274-291. doi:10.1007/ 978-3-642-23768-3_23. 2

[EB12] Elias M., Bezerianos A.: Annotating BI visualization dashboards: needs and challenges. In Proceedings of the SIGCHI Conference on Human Factors in Computing Systems (2012), Association for Computing Machinery, pp. 1641-1650. doi:10.1145/2207676.2208288. 1, 2,5

[emb21] Power bi embedded analytics, 2021. Accessed: 2021-11-22 URL: https://docs.microsoft.com/en-us/power-bi/developer/ embedded/embedded-analytics-power-bi. 9

[FDL20] Firat E. E., Denisova A., Laramee R. S.: Treemap Literacy: A Classroom-Based Investigation. Eurographics 2020 - Education Papers (2020), 10 pages. doi :10.2312/EGED . 20201032. 2

[FDPH17] Feng M., Deng C., Peck E. M., Harrison L.: HindSight: Encouraging Exploration through Direct Encoding of Personal Interaction History. IEEE Transactions on Visualization and Computer Graphics 23, 1 (2017), 351-360. doi:10.1109/TVCG . 2016. 2599058. 2

[Few21] FEw S.: There's nothing mere about semantics, 2021. Accessed 2021-12-2. URL: https://www. perceptualedge. com/blog/?p=2793.

[FWR*17] Federico P., Wagner M., Rind A., Amor-Amorós A., MiKsCH S., AIGNER W.: The Role of Explicit Knowledge: A Conceptual Model of Knowledge-Assisted Visual Analytics. In IEEE Conference on Visual Analytics Science and Technology (VAST '17) (2017), IEEE, pp. 92-103. doi: 10.1109/VAST. 2017.8585498. 2

[GIAM16] Gounder M. S., IYer V. V., Al MaZyad A.: A survey on business intelligence tools for university dashboard development. In $M E C$ Conference on Big Data and Smart City (ICBDSC '16) (2016), IEEE, pp. 1-7. doi:10.1109/ICBDSC. 2016.7460347. 1

[HDR*13] Hullman J., Drucker S., Riche N. H., LeE B., Fisher D., ADAR E.: A Deeper Understanding of Sequence in Narrative Visualization. IEEE Transactions on Visualization and Computer Graphics 19, 12 (2013), 2406-2415. doi:10/f5h287. 2

[HH] Holtz Y., Healy C.: From data to viz I find the graphic you need. Library Catalog: www.data-to-viz.com. URL: data-to-viz.com. 2

[hum21] Human resources, 2021. Accessed: 2021-11-22. URL: https://docs.microsoft.com/en-us/power-bi/create-reports/ sample-human-resources. 4

[KL16] Kwon B. C., LeE B.: A Comparative Evaluation on Online Learning Approaches using Parallel Coordinate Visualization. In Proceedings of the SIGCHI Conference on Human Factors in Computing Systems (2016), ACM Press, pp. 993-997. doi:10.1145/2858036.2858101. 2

[KPHH12] Kandel S., PaepcKe A., Hellerstein J. M., Heer J. Enterprise Data Analysis and Visualization: An Interview Study. IEEE Transactions on Visualization and Computer Graphics 18, 12 (2012), 2917-2926 doi:10.1109/TVCG. 2012.219. 2

[KPS03] Kang H., Plaisant C., Shneiderman B.: New Approaches to Help Users Get Started with Visual Interfaces: Multi-layered Interfaces and Integrated Initial Guidance. In Proceedings of the 2003 Annual National Conference on Digital Government Research (2003), dg.o '03, Digital Government Society of North America, pp. 1-6. 2,3

[Lau18] LAUREN: The 34 best interactive data visualizations from the new york times, 2018. Accessed: 2021-11-24. URL: https://getdolphins. 
com/blog/interactive-data-visualizations-new-york-times/. 1

[LGF12] Li W., Grossman T., Fitzmaurice G.: GamiCAD: a gamified tutorial system for first time autocad users. In Proceedings of the annual ACM symposium on User interface software and technology (2012), ACM Press, p. 103. doi:10.1145/2380116.2380131. 2

[LRIC15] LeE B., Riche N., Isenberg P., CARpendale S.: More Than Telling a Story: Transforming Data into Visually Shared Stories. IEEE Computer Graphics and Applications 35, 5 (2015), 84-90. doi : 10.1109/MCG. 2015.99. 2

[MLS*16] Morin R., LÉGer P.-M., Senecal S., BastaracheRoberge M.-C., Lefèbrve M., Fredette M.: The Effect of Game Tutorial: A Comparison Between Casual and Hardcore Gamers. In Proceedings of the Annual Symposium on Computer-Human Interaction in Play Companion Extended Abstracts (2016), ACM, pp. 229-237. doi : 10.1145/2968120.2987730. 2

[MM21] MA Q., Millet B.: Design Guidelines for Immersive Dashboards. Proceedings of the Human Factors and Ergonomics Society Annual Meeting 65, 1 (2021), 1524-1528. doi:10.1177/ 1071181321651177. 4

[PC05] PIROLli P., CARD S.: The sensemaking process and leverage points for analyst technology as identified through cognitive task analysis. In Proceedings of Conference on Intelligence Analysis (2005). 4

[pow21] Power bi embedded analytics client apis, 2021. Accessed: 202111-22. URL: https://docs.microsoft.com/en-us/javascript/ api/overview/powerbi/. 9

[QDRH19] QU Z., Du F., Rossi R. A., Howe B.: Aunt Lily Can Say Her Visualizations. In Proceedings of the Conference of the North (2019), Association for Computational Linguistics, pp. 4171-4186. doi : 10.18653/v1/N19-1423. 8

[Rib20] RIBECCA S.: The data visualisation catalogue, 2020. Accessed: 2021-11-19. URL: https://datavizcatalogue.com/. 2

[SCB*19] SARIKAya A., Correll M., Bartram L., Tory M., FISHER D.: What Do We Talk About When We Talk About Dashboards? IEEE Transactions on Visualization and Computer Graphics 25, 1 (2019), 682-692. doi :10.1109/TVCG. 2018.2864903. 1, 2, 8, 10

[SGP*19] Stoiber C., Grassinger F., Pohl M., Stitz H., Streit M., Aigner W.: Visualization Onboarding: Learning How to Read and Use Visualizations. preprint, Open Science Framework, 2019. doi: 10.31219/osf.io/c38ab. 1, 2, 3

[SH10] SEgEL E., HeER J.: Narrative Visualization: Telling Stories with Data. IEEE Transactions on Visualization and Computer Graphics 16, 6 (2010), 1139-1148. doi:10.1109/TVCG.2010.179. 2

[SMWH17] Satyanarayan A., Moritz D., Wongsuphasawat K., HEER J.: Vega-Lite: A Grammar of Interactive Graphics. IEEE Transactions on Visualization and Computer Graphics 23, 1 (2017), 341-350. doi : 10.1109/TVCG.2016.2599030. 2, 3, 4

[SSX*21] Shi D., Sun F., Xu X., Lan X., Gotz D., CaO N.: AutoClips: An Automatic Approach to Video Generation from Data Facts. Computer Graphics Forum 40, 3 (2021), 495-505. doi : 10.1111/cgf. 14324. 2

[sup21] Supplementary material for dashboard onboarding, 2021. Accessed: 2021-12-2. URL: https://osf. io/zsb5a/. 3, 5, 6, 9

[SWG*21] Stoiber C., WAlchshofer C., Grassinger F., Stitz H., Streit M., Aigner W.: Design and comparative evaluation of visualization onboarding methods. pp. 1-5. doi:10.1145/3481549. 3481558. 1, 2

[tab21] Tableau, 2021. Accessed: 2021-11-22. URL: https://www. tableau.com/. 9

[TBFGC21] Tory M., Bartram L., Fiore-Gartland B., Crisan A.: Finding Their Data Voice: Practices and Challenges of Dashboard Users. IEEE Computer Graphics and Applications (2021), 15. 2, 8
[THhS16] Tschinkel G., Hafner R., Hasitschka P., SAbol V.: Using Micro-Visualisations to Support Faceted Filtering of Recommender Results. In Conference Information Visualisation (IV) (2016), IEEE, pp. 318-323. doi:10.1109/IV.2016.77. 8

[TLM16] Tanahashi Y., Leaf N., Ma K.: A Study On Designing Effective Introductory Materials for Information Visualization. Computer Graphics Forum 35, 7 (2016). doi : 10.1111/cgf. 13009. 1, 2

[Tra21] Training D. B. P. B.: Sales analysis, 2021. Accessed: 202111-22. URL: https://www. youtube. com/watch?v=XceUqw7kVmc\&t= 198s\&ab_channel=DataBearPowerBITraining. 6

[VIGPT19] VÁZQueZ-Ingelmo A., García-Peñalvo F. J., Therón R.: Capturing high-level requirements of information dashboards' components through meta-modeling. In Proceedings of the Conference on Technological Ecosystems for Enhancing Multiculturality (2019), ACM, pp. 815-821. doi: 10.1145/3362789.3362837. 2, 3, 4

[WLF*19] WANG Q., Li Z., FU S., CUI W., QU H.: Narvis: Authoring Narrative Slideshows for Introducing Data Visualization Designs. IEEE Transactions on Visualization and Computer Graphics 25, 1 (2019), 779788. doi : 10.1109/TVCG. 2018.2865232. 2

[Won20] WONGSUPHASAWAT K.: Encodable: Configurable grammar for visualization components. In IEEE Visualization Conference (VIS '20) (2020), IEEE, pp. 131-135. doi :10.1109/VIS47514.2020.00033. 9

[WSC17] WeXler S., ShafFer J., COTGReave A.: The big book of dashboards: visualizing your data using real-world business scenarios. Wiley, 2017. 1

[WSMrb20] Wang Z., Sundin L., Murray-Rust D., BaCh B.: Cheat Sheets for Data Visualization Techniques. In Proceedings of the CHI Conference on Human Factors in Computing Systems (2020), ACM, pp. 1-13. doi:10.1145/3313831.3376271. 2 